\title{
Discussion
}

\section{Alzheimer Research Forum Live Discussion: Meet New Players, Histone Deacetylase and Sirtuin - Will They Help the Cell Cycle, DNA Repair, and Gene Expression Break Into Alzheimerology's Major League?}

\author{
(http://www.alzforum.org/res/for/journal/transcript.asp?LiveID=172)
}

Participants: Craig Atwood (University of Wisconsin), Greg Brewer (Southern Illinois University School of Medicine), Karl Herrup (Rutgers University), Vikram Khurana (Massachusetts General Hospital), Dohoon Kim (Picower Institute of MIT), Bruce Lamb (Cleveland Clinic), Brett Langley, Melanie Leitner (Prize4Life), Virgil Muresan (New Jersey Medical School), Rachael Neve (Picower Institute of MIT), Kevin Park (University of British Columbia), Holger Patzke (EnVivo Pharmaceuticals, Inc.), Gabrielle Strobel (Alzheimer Research Forum), Li-Huei Tsai (Picower Institute of MIT), Bruce Yankner (Harvard Medical School).

Note: Transcript has been edited for clarity and accuracy.

Gabrielle Strobel: In the journal Neuron, researchers led by Li-Huei Tsai at MIT's Picower Institute reported results from their $\mathrm{p} 25 / \mathrm{Cdk} 5$ mouse model of neurodegeneration. The data suggest that aging-related or ADrelated stress factors, by way of generating p25, inhibit the normal function of the chromatin-modifying enzyme histone deacetylase 1 (HDAC1) in adult neurons. This dysregulation of HDAC1 was reported to cause both cell cycle activity and DNA breaks, and it does so long before neurons degenerate, these investigators found.

I invite all panelists to state briefly, if they can, how Dohoon and Li-Huei's latest findings [1] fit in, or not, with their own data and put a question to the presenters.

Karl Herrup: I can start with the role of Cdk5 activity itself. The role of p25 is quite clear and the inhibitor data with the luciferase assay is compelling, but since p25 binds directly to histone deacetylase 1 (HDAC1), do we really need the kinase?

Li-Huei Tsai and Dohoon Kim: Karl, based on the widely reported neuroprotective effect of Cdk5 inhibition, as well as a subset of our results (e.g., showing that inhibition of HDAC 1 transcription repressor activity by p25 requires Cdk5 activity), we feel it is most likely that Cdk5 activity is required. Our preliminary findings suggest that the underlying mechanism is not direct phosphorylation of HDAC1 by Cdk5. Rather, we have preliminary evidence that suggests that $\mathrm{p} 25 / \mathrm{Cdk} 5$ interaction with HDAC1 allows phosphorylation of a corepressor for HDAC1 that ultimately results in HDAC1 inactivation. 
Vikram Khurana: Dohoon, Li-Huei, congratulations on the great paper! Cdk5 seems to be a central player in mediating neurodegeneration in a number of paradigms and I am not very familiar with the Cdk5 literature. But in looking through the citations you give in your paper, I note that many of the examples demonstrate a role for $\mathrm{Cdk} 5 / \mathrm{p} 25$ by gain, rather than loss, of function. I am wondering if you have looked in your in vivo stroke model (or other in vivo, in vitro models) in a genetic loss-of-function background (e.g., p35 null or conditional knockout mice) to determine the relative role of $\mathrm{Cdk} 5$ in mediating neurodegeneration?

Li-Huei Tsai and Dohoon Kim: Vikram, we are aware of many studies that show that inhibition of Cdk5 using pharmacological inhibitors or DNK5 can be neuroprotective, for example, in ischemia. Furthermore, there is much evidence for a protective effect of calpain inhibitors, which should inhibit p25 generation. But it should be noted that calpain inhibition will inhibit proteolysis of the other calpain targets as well. There are a few studies showing neuroprotection in the p35 knockout.

Rachael Neve: Li-Huei, I would be interested to know your interpretation of why you found activation of an HDAC protective, whereas in other neurodegenerative models that have been published, inhibition of HDACs has been protective.

Li-Huei Tsai and Dohoon Kim: Rachael, this is an important question. This result was initially perplexing to us when considering that many laboratories, including ours, have convincingly demonstrated that general HDAC inhibitors can have various benefits in the central nervous system (CNS). We have tried to address this (in length) in the discussion section of our manuscript. In short, the emerging picture we are getting at is that inhibition of certain HDACs result in beneficial gene expression, while inhibition of others, such as HDAC1, leads to detrimental effects such as expression of cell cycle genes. Our laboratory recently identified one of these "beneficial" targets of HDAC inhibitors, which will be revealed shortly.

Craig Atwood: What physiological signals regulate HDAC1 expression?

Li-Huei Tsai and Dohoon Kim: Craig, so far we have only looked in the context of p25-induced HDAC1 inhibition. From prior studies from our laboratory and oth- ers, a variety of neurotoxic stimuli such as excitotoxicity, oxidative stress, amyloid fibril treatment, etc., are able to generate $\mathrm{p} 25$, so these would be good contexts to examine HDAC1 depression.

Virgil Muresan: Hello, Li-Huei; very nice work. Do you know if p25 binds to other HDACs, like HDAC6? How related are the different HDACs?

Li-Huei Tsai and Dohoon Kim: Virgil, we have found p25 does bind other HDACs and are currently exploring this.

Virgil Muresan: I am impressed with the many important roles of protein acetylation. I am also referring to this month's paper by Creppe et al. [2] on the role of tubulin acetylation in neuronal migration and differentiation (a topic dear to Li-Huei, too). How extensive is acetylation of proteins in neurons; i.e., how many proteins are acetylated? Should we regard acetylation as we regard phosphorylation?

Greg Brewer: Li-Huei, your nice work also could be interpreted as a failed attempt at repair. Maybe there is some room for less overexpression or more moderate HDAC1 expression. Have you done any dose-response studies to see the shape of the curve?

Li-Huei Tsai and Dohoon Kim: Greg, yes, we definitely should consider the possibility of failed repair. Interestingly, as we briefly showed in the paper, if you turn on p25 for two weeks, then turn it off for four weeks, then the robust DNA damage that you see at two weeks appears completely gone, without any neuronal loss. The neurons do appear to have capacity to repair DNA damage, but beyond a certain point, death ensues.

Greg Brewer: Li-Huei, that is comforting to hear that all is not lost and there is more hope for reversibility. You studied the in vitro effects on embryonic neurons. We see some reversibility of $\mathrm{A} \beta$-killing by a deacetylase inhibitor in old rat neurons in vitro.

Li-Huei Tsai and Dohoon Kim: Greg, that is certainly interesting. Thanks for offering this information.

Karl Herrup: Li-Huei, the co-repressor idea would be a neat twist. The two weeks on/four weeks off story of DNA repair is also interesting. Both DNA damage and cell cycle protein expression return to baseline. A couple of thoughts: The first is that this is not what we 
see with the cell cycle events in the AD models. Even if we remove the stimulus, the cycle proteins stay on. The second is that if all of this is really reversible after two weeks, what is it that pushes the neuron over the edge to finally die?

Bruce Lamb: Excellent presentation, Li-Huei. One of the things I noticed is that although you state that there is no gliosis at the timepoint examined, a number of the genes on the microarray exhibiting alterations include "inflammatory" genes including GFAP, class II MHC, etc., in addition to the cell cycle/repair genes. Do you think the glial response is playing a role in the neurotoxicity?

Li-Huei Tsai and Dohoon Kim: Bruce Lamb, yes, at two weeks of p25 expression, there are no signs of gliosis according to immunohistochemistry. Signs of neuronal death are not seen until a few weeks later. But, perplexingly, we did note that some inflammatory genes were found to be upregulated in the microarray. In short, we cannot completely rule out the role of inflammation in the p25 mouse, but that is something we have not really looked into yet.

Karl Herrup: Li-Huei, with the inflammation factoid, you may just have answered my question about what finally pushes the neuron to die.

Gabrielle Strobel: Some feedback on inflammation from the human front: Chet Mathis and Bill Klunk published this month in Archives of Neurology work where they imaged the brains of people with $\mathrm{MCI} / \mathrm{mild} /$ moderate AD both with PIB PET for amyloid and a microglial activation PET marker. The microglial signal was not different between the groups, suggesting either that microglial activation is not an early marker of AD or that this particular tracer is insensitive [3].

Karl Herrup: Gabrielle, we only find early inflammatory markers upregulated. I would be willing to bet that the imaging study used a late marker, which we, too, find very late in the disease process (well after cell cycle initiation).

Gabrielle Strobel: Karl, this was the C11-labeled RPK11195 that indicates microglial activation. If only there were more PET tracers for more inflammation markers!
Li-Huei Tsai and Dohoon Kim: Gabrielle, very interesting point. In our p25 mice, in terms of immunohistochemistry, we see reactive gliosis around the same time as neuronal death.

Gabrielle Strobel: Li-Huei, given how powerful PET can be, it is really a huge gap that we have so few labels. Nothing for tau, for $\alpha$-synuclein, for synaptic activity markers ....

Melanie Leitner: And even the PET labels that we do have are not very good (but at least the AD field has PIB while ALS has pretty much nothing).

Bruce Yankner: Li-Huei, which do you think comes first - DNA damage or cell cycle activation? As you know, DNA replication can convert incipient strand breaks into double-strand breaks.

Vikram Khurana: Bruce Yankner, it is interesting to note that ectopic cyclin expression in the Drosophila brain is sufficient to induce phosphorylation of $\mathrm{H} 2 \mathrm{AX}$, so I do think the epistasis of cell cycle-DNA damage is complex and potentially bidirectional. Just as in cycling cells, it seems that forcing cell cycle upon postmitotic neurons will induce double-stranded DNA breaks.

Li-Huei Tsai and Dohoon Kim: Bruce Yankner, great comment. There is potential for cell cycle activation to facilitate DNA damage like you mentioned. Conversely, Krumann/Mattson's study showed that DNA damage can trigger cell cycle activation as well. So there is some potential interplay between cell cycle reentry and DNA damage aside from our showing that HDAC1 inhibition may be a common trigger for both. I should note that we did try to look at earlier periods of p25 induction and could not find a time where one appears before the other. So they are definitely tightly correlated.

Brett Langley: Li-Huei, great talk. Did you ever look at DNA damage by comet after HDAC1 inhibition/knockdown (in addition to the H2AX)?

Li-Huei Tsai and Dohoon Kim: Brett, no we have not looked at that.

Kevin Park: Thank you for a very informative presentation. My question to you is that when you treat wildtype mice with HDAC1 (injected IP), is neurodegeneration observed following $\gamma \mathrm{H} 2 \mathrm{AX}$ induction? [Editor's 
note: $\mathrm{H} 2 \mathrm{AX}$ induction is a histone, $\gamma$ its phosphorylated form.]

Li-Huei Tsai and Dohoon Kim: Kevin Park, we did note that a subset of the H2AX positive neurons were cleaved caspase 3 positive, indicating that they may be beginning to undergo neurodegeneration, but we actually have not looked at later points of treatment in enough detail to confidently address this.

Holger Patzke: Li-Huei and Dohoon, as you know, we are developing HDAC inhibitors for CNS disease. How do you fit in data from several laboratories showing that HDAC inhibition is completely protective (even post-damage dosing) in the rat middle cerebral artery occlusion (MCAO) model?

Bruce Lamb: Holger, I was unaware of the data on HDAC inhibition in stroke. Would this also suggest potentially a role of HDACs in other pathways induced upon stroke? What are these pathways?

Holger Patzke: I do not remember all the details, but a bcl-2 and kinase signaling link among others was demonstrated.

Li-Huei Tsai and Dohoon Kim: Very good, important point. Yes, there is definitely good data for neuroprotection by HDACi in MCAO. Maybe Brett can comment on this better, but he had a great talk at the last Society for Neuroscience meeting showing that even though there is good neuroprotection against acute neurotoxic stress by HDACi, there is also a baseline toxicity that is observed for most of these drugs when given for longer periods or higher doses. He presented that when a specific inhibitor of HDAC6 was given, he could get the same neuroprotection without the baseline neurotoxicity. Again, this is getting to the idea that there are certain HDACs responsible for the benefits, and that certain HDACs you do not want to inhibit too much or too long or you will get detrimental effects.

Brett Langley: Holger, to comment further on what Li-Huei was saying, we have found that the duration of pan-HDAC inhibitor exposure is critical. The toxicity can be abrogated (while neuroprotection sustained) by short duration treatment (two- to three-hour treatment in neurons in culture). Given this finding, perhaps treating in vivo (such as in stroke) is more analogous to a pulse-type treatment and consistent with protection. Li-Huei, did you ever try a pulse treatment with MS275 in your experiments?
Li-Huei Tsai and Dohoon Kim: Brett, no we did not, but good suggestion. Your study on the pulse treatment in HDACi was a great read. I think it is definitely worth noting/having caution that prolonged and highdose treatment with HDAC inhibitors can be risky, and in the future more specific knowledge and more specific inhibitors should be very fruitful.

Holger Patzke: Brett, that is very interesting. In fact, most HDAC inhibitors have that kind of pharmacokinetics (PK) profile in vivo, and this of course differs greatly from herpes simplex virus (HSV)-mediated overexpression.

Gabrielle Strobel: To the representatives from biotech /pharma companies that target HDACs, can either of you share experiences with HDAC inhibition versus activation? Are inhibitors of specific HDACs, and activators of others, in hand, or are the current compounds hitting multiple HDACs?

Holger Patzke: Gabrielle, select HDACi are available for a few HDACs (HDAC6). The bulk of the work has been done in cancer, and little in vivo data are available regarding the CNS. Most HDACi are promiscuous.

Gabrielle Strobel: Holger, are there any HDAC activators? Or molecules to inhibit a physiological HDAC1 repressor?

Vikram Khurana: I know of one paper by David Park in PNAS where he uses a knockdown approach to show Cdk5 plays a role in excitotoxic, rather than delayed, cell death in hypoxia, including an in-vivo stroke model [4]. In the latter case, his data indicate a more central role for $\mathrm{Cdk} 4$. After reading the paper, I wondered if multiple upstream mediators might lead to the various neurodegenerative processes you show (double-strand breaks, chromatin changes, cell cycle activation) in different contexts?

Greg Brewer: All, one concept for a mechanism of cell death is exhaustion of redox energy. We find an oxidized redox potential in old rat neurons that makes them more susceptible to glutamate and $\mathrm{A} \beta$ toxicity [5].

Li-Huei Tsai and Dohoon Kim: Karl, interesting points. I would like to say that the model we have of turning on and off p25 at will is great for experimentally getting at some issues, but probably the abrupt taking away of p25 that we did would not be applica- 
ble to what is going on chronically, over a long time, in $\mathrm{AD}$, and that could account for these findings you mentioned.

Karl Herrup: Li-Huei and Kevin Park, fascinating. Death is an analog function, not digital. We find the same thing: Caspase 3 activation in perfectly healthy looking neurons. I am willing to bet it is reversible, too.

Gabrielle Strobel: Li-Huei and Dohoon, this came up in Agata Copani's written comment prior to the discussion as well. Would you like to address her question about whether cell cycle re-entry kills the neurons or is incidental to the death process?

Li-Huei Tsai and Dohoon Kim: Gabrielle, regarding Agata Copani's comment whether cell cycle re-entry is required or incidental for neurodegeneration: Dr. Copani has provided many insightful comments on the Alzforum site regarding our study, much of which we agree with or postulate to be accurate. One point I would like to make, though: I feel our study does not suggest that cell cycle reactivation is in some way incidental and marginal in the death process. In our study, we show that DNA damage and cell cycle reactivation appears before neuronal death, and at later periods of p25 induction, is closely associated with neuronal death. So, like she commented, the relative degrees of contribution of the two processes have not been nailed down. We feel, as do many in the field, that cell cycle does play a critical role in neuronal death. With the p25 model, which concomitantly displays both cell cycle re-entry and DNA damage, we are in an excellent position to test the relative contribution of each to neuronal death. It may well be that both are required to trigger death - the cell cycle reactivation to turn on a cell cycle-dependent checkpoint, and the DNA damage to trigger it and induce neuronal death - as proposed previously by Dr. Hanawalt and colleagues.

Vikram Khurana: Li-Huei and Dohoon, you show a prominent checkpoint transcriptional response to p25 overexpression. Have you tried to block cell cycle in your p25-overexpressing in vivo model to show cell cycle activation mediates neuronal death?

Li-Huei Tsai and Dohoon Kim: Vikram, no we have not yet. We expect that if we do so we may get the same cell cycle re-entry and DNA damage but rescue against death. It would be a good experiment.
Gabrielle Strobel: Li-Huei and Dohoon, regarding your emerging hypothesis of age-related or epigenetic deregulation of HDAC 1 as causing neurodegeneration: You know that in Alzheimer's in particular, amyloid- $\beta$ is still a central focus of attention. You have worked on it quite a bit yourselves. How does $\mathrm{A} \beta$ fit into your hypothesis? As an AD-specific upstream trigger of calpain, among other triggers, for the cascade you describe? Or what else?

Li-Huei Tsai and Dohoon Kim: Gabrielle, great comment regarding $\mathrm{A} \beta$. We do see $\mathrm{A} \beta$ upregulation in the p25 mouse, so definitely it is possible. But I know Bruce Yankner has important data/information/insights into this idea and I would like to refer this question to him.

Bruce Yankner: Gabrielle, I am not sure if $\mathrm{A} \beta$ is a cause or a result of DNA damage, although I suspect both may be true. A central question is what starts it all, which I doubt is $\mathrm{A} \beta$.

Virgil Muresan: Li-Huei and Dohoon, how do you think that $\mathrm{A} \beta$ becomes elevated in the $\mathrm{p} 25-\mathrm{GFP}$ mouse? Is this due to $\mathrm{A} \beta \mathrm{PP}$ phosphorylation?

Li-Huei Tsai and Dohoon Kim: Virgil, we think, as we showed in a previous study, this should involve upregulated BACE1. Karen Duff later also showed upregulated BACE1 in p25 mice, as a result of transcriptional upregulation involving STAT3. But, certainly, phosphorylation of $\mathrm{A} \beta \mathrm{PP}$ by $\mathrm{p} 25$ may have an important role in $\mathrm{A} \beta \mathrm{PP}$ processing and account for what we see.

Gabrielle Strobel: Bruce Yankner, do your age-related gene expression changes together point to any particular upstream process?

Bruce Yankner: Our expression data suggest that normal aging of the human cortex is accompanied by increased expression of the cyclin-dependent kinase inhibitor p57/kip2 and reduced expression of the Cdk5 activators (p35 and p39). These are the major changes observed in cell cycle genes, which are also detected to a lesser extent in the aging mouse brain. These changes would be expected to inhibit, not activate, cell cycle progression. However, this might change in AD.

Kevin Park: Li-Huei and Dohoon, I think the evidence for the toxic nature of p25 is clear. But p25 can wreak 
havoc in so many ways. In primary neuron culture you were able to rescue by HDAC 1 expression. However, there is significant disparity between culture system and in vivo. In your opinion, how much of the degenerative phenotype in your conditional knockout mice is due to p25-mediated HDAC inhibition?

Virgil Muresan: Li-Huei and Dohoon, is it still possible that what you see in your study is also due to some other effects of p25 overexpression? Maybe, as you said, on other HDACs in the cytoplasm?

Li-Huei Tsai and Dohoon Kim: Kevin and Virgil, yes, the p25 mouse has many phenotypes aside from DNA damage and cell cycle reactivation - amyloidrelated pathology, tau pathology, synaptic pathology, cognitive decline. We are just beginning to look at the various aspects.

Gabrielle Strobel: How about humans? How can we build on the 25 overexpression models to address if a cascade of age-related stressor-p25 induction-HDAC deregulation-cycle/DNA breaks-degeneration actually happens in people? Are there markers we could trace in CSF, for example? p25 itself?

Li-Huei Tsai and Dohoon Kim: Gabrielle, I like your thinking about how we can better trace this in humans. I am all ears to suggestions.

Gabrielle Strobel: Li-Huei, I am out of my depth to make specific technical suggestions, but CSF markers have become quite fruitful as AD diagnostic aids, in research studies as a predictive tool, and increasingly as biomarkers in drug trials. Even though they are not perfect: $\mathrm{A} \beta$ is a body-wide protein, tau is an intraneuronal protein, BACE1 apparently goes up in CSF even as $\mathrm{A} \beta$ goes down in CSF. Considering that all of these are far from clear-cut but useful in human research even so, I would think that any markers of DNA damage, HDAC deregulation, p35 cleavage would be worth exploring initially, no?

Li-Huei Tsai and Dohoon Kim: Gabrielle, definitely. It would be interesting to get at CSF markers for DNA damage, p35 cleavage, etc.

Gabrielle Strobel: Dear Li-Huei and Dohoon, I want to thank you very much on behalf of Alzforum and our audience for your time and openness in this hour.

Li-Huei Tsai and Dohoon Kim: Thank you very much, everyone, for your helpful inputs.

Citations: Add the Tsai reference and move all the numbers of the other citations down:

\section{REFERENCES}

[1] Kim D, Frank CL, Dobbin MM, Tsunemoto RK, Tu W, Peng PL, Guan J-S, Lee B-H, Moy LY, Giusti P, Broodie N, Mazitschek R, Delalle I, Haggarty SJ, Neve RL, Lu Y, Tsai LH (2008) Deregulation of HDAC 1 by $\mathrm{p} 25 / \mathrm{Cdk} 5$ in neurotoxicity. Neuron 60, 1-15.

[2] Creppe C, Malinouskaya L, Volvert ML, Gillard M, Close P, Malaise O, Laguesse S, Cornez I, Rahmouni S, Ormenese S, Belachew S, Malgrange B, Chapelle JP, Siebenlist U, Moonen G, Chariot A, Nguyen L (2009) Elongator controls the migration and differentiation of cortical neurons through acetylation of alpha-tubulin. Cell 136, 551-64.

[3] Wiley CA, Lopresti BJ, Venneti S, Price J, Klunk WE, DeKosky ST, Mathis CA (2009) Carbon 11-labeled Pittsburgh Compound B and carbon 11-labeled (R)-PK11195 positron emission tomographic imaging in Alzheimer disease. Arch Neurol 66, 60-7.

[4] Rashidian J, Iyirhiaro G, Aleyasin H, Rios M, Vincent I, Callaghan S, Bland RJ, Slack RS, During MJ, Park DS (2005) Multiple cyclin-dependent kinases signals are critical mediators of ischemia/hypoxic neuronal death in vitro and in vivo. Proc Natl Acad Sci U S A 102, 14080-5.

[5] Parihar MS, Brewer GJ (2007) Mitoenergetic failure in Alzheimer disease. Am J Physiol Cell Physiol 292, C8-23. 